\title{
Trivium
}

Revue franco-allemande de sciences humaines et sociales - Deutsch-französische Zeitschrift für Geistesund Sozialwissenschaften

$10 \mid 2012$

Lisibilité

\section{Die Traumdeutung im antiken Mesopotamien}

\author{
Jean Bottéro
}

Traducteur : Andreas Pfeuffer

\section{OpenEdition}

Journals

Édition électronique

URL : http://journals.openedition.org/trivium/4190

DOI : 10.4000/trivium.4190

ISSN : 1963-1820

Éditeur

Les éditions de la Maison des sciences de l'Homme

Référence électronique

Jean Bottéro, «Die Traumdeutung im antiken Mesopotamien », Trivium [Online], 10 | 2012, online erschienen am 30 März 2012, abgerufen am 07 September 2020. URL : http://

journals.openedition.org/trivium/4190; DOI : https://doi.org/10.4000/trivium.4190

Ce document a été généré automatiquement le 7 septembre 2020.

\section{(c) (i) () $\Theta$}

Les contenus des la revue Trivium sont mis à disposition selon les termes de la Licence Creative Commons Attribution - Pas d'Utilisation Commerciale - Pas de Modification 4.0 International. 


\title{
Die Traumdeutung im antiken Mesopotamien
}

\author{
Jean Bottéro \\ Traduction : Andreas Pfeuffer
}

\section{NOTE DE L'ÉDITEUR}

Copyright Françoise Bottéro, Alain Bottéro.

Wir danken Françoise Bottéro und Alain Bottéro für die freundliche Genehmigung, diesen Artikel zu übersetzen.

1 Im Unterschied $\mathrm{zu}$ anderen Zivilisationen wie der Ägyptens unter den Pharaonen stellten die Erkundung, die Praxis und die Deutung von Träumen für die Weissagung im antiken Mesopotamien nur einen bescheidenen Ausschnitt eines viel weiter reichenden Unternehmens dar, dessen Gegenstandsbereich sich letztlich mit dem gesamten diesseitigen Universum deckte. In den Augen dieser Menschen hatte alles auf der Welt wahrsagerische Bedeutung, die Träume wie alles Sonstige auch. Und genau deshalb erscheint es mir angebracht, zunächst dieses allumfassende Weissagen in groben Zügen darzustellen, um dann darin die Traumdeutung umso leichter und ergiebiger verorten zu können. ${ }^{1}$

Doch um einen Zugang zur Sicht der alten Mesopotamier auf diese Dinge zu bekommen, ist es unerlässlich, kurz einige Grundparameter ihres Denksystems in Erinnerung zu rufen. Sie waren davon überzeugt, dass die sie umgebende Welt ihren Daseinsgrund nicht in sich selbst hatte. Sie hing vielmehr gänzlich von überirdischen Mächten ab, die sie geschaffen hatten und die über sie herrschten, und dies zu allererst zu ihrem eigenen Vorteil. Sie stellten sich diese Götter nach dem Bild der Menschen vor, ihnen jedoch vollkommen überlegen aufgrund ihres ewigen Lebens, ihrer Intelligenz und ihrer die unsrige unendlich übersteigende Macht. Alles hienieden, die Wesen wie die Geschehnisse, unterlag also dem Walten und Wollen der Götter und fügte sich ein in eine Art Generalplan, den sie im Kopf hatten, selbst wenn er als solcher für die 
Menschen nicht zu durchschauen war, weil sie dessen Ablauf nur von Tag zu Tag entdeckten. Nichts von dem, was uns hinsichtlich der Vergangenheit, der Gegenwart und natürlich der Zukunft verborgen bleibt, entging ihnen und ihrer Entscheidung. Doch stand es ihnen frei, die Menschen nach Gutdünken darüber in Kenntnis zu setzen. Darin liegt die ganze Bedeutung des Weissagens.

Wie konnte es zu einer solchen Mitteilung kommen? Auf direktem oder indirektem Wege. Der direkte Weg bestand für die Götter darin, ohne Umschweife zu offenbaren, was sie zu sagen hatten. Belegt sind nur ein oder zwei solcher öffentlichen offenbarungen (die Armee: Streck [1975], S. 48 ff. und S. 95 ff.), offenbar zogen die Götter lieber einen einzigen Vermittler, ein »Medium« heran, um diesem ihr Geheimnis anzuvertrauen und ihn mit dessen Verbreitung zu betrauen. Anscheinend konnte egal wer ausgewählt werden und mittels einer auditiven oder visuellen Verbindung oder bevorzugt auch beider zugleich von einem übernatürlichen Wesen dessen Botschaft erhalten, ob es nun um die Vergangenheit, die Gegenwart oder vor allem um die Zukunft ging. Der Inhalt solcher Offenbarungen war zumeist klar und unmittelbar einsichtig, doch konnte er auch dunkel sein, und dann war eine besonderen Fachleuten vorbehaltene Auslegung unerlässlich. Diesen ersten Typ von Mantik, der der mündlich weitergegebene direkten Rede nachgebildet war, können wir angesichts seines übernatürlichen Kontexts als inspirierte Weissagung bezeichnen. Unserem Wissen nach war er im eigentlichen Mesopotamien wohl weder allzu verbreitet - oder gar verallgemeinert - noch sonderlich geschätzt, sieht man einmal von einigen wenigen Epochen und Milieus ab.

Der andere, »indirekte« Typ von Mantik, den ich deduktive Weissagung nenne und der, urteilt man nach den zahllosen Texten, die uns überliefert sind, sehr viel weiter verbreitet war, beruht auf dem Modell der schriftlichen Mitteilung. Die Götter kommunizierten nicht »mündlich", was sie zu sagen hatten, sie kodierten es graphisch, verschriftlichten es, um es der Lektüre ihrer Empfänger zu unterbreiten. Das verlangt nach einiger Erklärung. ${ }^{2}$ Man darf nicht vergessen, dass die alten Mesopotamier, die wahrscheinlich um 3000 vor Christus die älteste bekannte Schreibmethode erfunden hatten, von dieser Neuerung durch und durch beeindruckt und geprägt waren, und dies nicht nur, weil diese tiefgreifende Transformation sie der schriftlichen Überlieferung unterstellte ${ }^{3}$, sondern auch, weil sich ihr eigenes graphisches System in gewissem Sinne in ihr Denken einlagerte und es formte. Diese Schrift war im ursprünglichen Zustand (und ist es mehr oder weniger zum Teil geblieben) ganz und gar piktographisch, das heißt, sie evozierte die auszudrückenden Dinge durch sie repräsentierende Zeichnungen, was direkt (die Ähre stand für »Getreide«; das Schamdreieck für »die Frau«) oder indirekt (die Gebirgsumrisse für »das Land" oder »das Ausland«; der Fuß für "stehen «, "gehen« oder »mitnehmen«, »mitreißen« ...) geschehen konnte. Kurz gesagt, stellte man die Dinge durch diese anderen Dinge in Form von Umrisszeichnungen dar. Diese Methode hat die Vorstellungskraft der alten Mesopotamier geprägt, sie hat ihrer "Logik« eine Anzahl von Schemata geliefert (vgl. weiter unten), darunter jenes, das gewissermaßen einen Typus von Weissagung rationalisiert hat. Dessen »objektive« Grundlage war sicherlich die wiederholte Beobachtung von Ereignisabläufen gewesen: Ein Ereignis etwa, das aufgrund seiner Ungewöhnlichkeit Aufmerksamkeit erregte und einem anderen, ebenfalls zufälligen und unerwarteten Ereignis vorausging, wurde als Vorzeichen des Letzteren aufgefasst, ungeachtet dessen, ob ihr Zusammenhang nun tatsächlich oder eingebildet war. Beispielweise konnte eine bestimmte wenig geläufige meteorologische Erscheinung eine Katastrophe für die Landwirtschaft einleiten; die Erscheinung einer Missbildung ließ ein Unglück befürchten, das ungewöhnliche 
Verhalten eines Menschen oder Tieres konnte den Auftakt für eine Wendung im Leben der Menschen bilden. Hier genau kam das Schema der Schrift zum Zuge, insofern die ankündigenden Erscheinungen als von ihren Urhebern, den Göttern, bewirkte Dinge aufgefasst wurden, deren Ziel es war, andere Dinge zu bedeuten. "Göttliche Piktogramme« gleichsam zur Übermittlung einer von oben kommenden Botschaft betreffs dessen, was sich in der Folge ereignen sollte. Nachdem der Rahmen für diesen Typ von »Vorher-Sage« geschaffen war, vielleicht weil er dem auf Präzision, Klassifikation und Rationalität bedachten Wesen der alten Mesopotamier entsprach, entwickelten sie sie in einem außergewöhnlichen Grad fort und systematisierten sie. Auf diese Weise bildete sich bei ihnen, wie für die Schrift auch, eine Art »Code« heraus, dank dessen die mit den Bedeutungen der verschiedenen göttlichen Piktogramme vertrauten Spezialisten (im Gegensatz zu der von der inspirierten Weissagung nicht zu lösenden Schwammigkeit und Ungewissheit) genauestens und eindeutig deren Botschaft bezüglich der Zukunft entschlüsseln konnten. Sie konnten sie daraus ableiten - deshalb auch die Bezeichnung deduktive Weissagung. Und weil ja das gesamte Universum von den Göttern, die dessen Funktionieren und Lauf bestimmten, auf Gedeih und Verderb abhing, sah man logischer Weise die irdische Welt in ihrer Gesamtheit als Stütze ihrer als solcher verstandenen "Schrift« und als Träger ihrer zu entziffernden Botschaften. Über diesen Umweg wurde die Natur insgesamt mit divinatorischer Bedeutung ausgestattet: die Bewegungen der Sterne und das Auftauchen von Meteoren; der Lauf der Zeit; die Auffälligkeiten der mineralen und pflanzlichen Welt; das Aussehen und das Verhalten der Lebewesen (vor allem bei der Geburt oder im Augenblick des Todes), mehr noch der Menschen: die besonderen Eigenheiten ihrer Physiognomie und ihre Verhaltensgewohnheiten; und schließlich ihr Traumleben.

5 Um besser erfassen zu können, wie Letzteres sich in ein universelles Weissagungssystem hat einfügen können, sollten wir uns das Wenige, das wir von der Vorstellung wissen, die sich die alten Sumerer und Babylonier von der Welt der Träume machten, in Erinnerung rufen. Nehmen wir einmal an, sie hatten eine klare Vorstellung davon - was durchaus zweifelhaft ist -, so haben sie sich doch nie die Mühe gemacht, sie uns zu erklären, und wir müssen sie uns selbst erschließen, und zwar sowohl aus ihrem Vokabular als auch aus der Art und Weise, in der sie über ihre Träume sprechen.

6 Die Bezeichnungen, die sie ihnen gaben, belegen, dass sie sie im Wesentlichen mit der Nacht (auf sumerisch ma-mú(d) - nach A. Falkenstein - und máš-ge $e_{6}$ : Produkt der Nacht $\aleph^{4}$, und auf akkadisch tabrit mûši: "Vision der Nacht«) und mit dem Schlaf (das akkadische šuttu: "Schlaf« hat dieselbe "Wurzel« *wšn - vgl. hebräisch yšn und arabisch wsn: "schlummern« - wie šittu: "Schlaf«) in Verbindung brachten. Der Traum war also ein im Wesentlichen nächtliches Phänomen und vor allem dem Schlaf eigen (obgleich er zufällig war, da man auch traumlosen Schlaf kannte). Trotzdem stellte das "Träumen" weder eine Handlung noch einen spezifischen Zustand dar. Weder im Sumerischen noch im Akkadischen gibt es ein entsprechendes Verb. Man sagte nur einen Traum "sehen« (akk. amâru und naţâlu; manchmal naplusu und šubrû), und der Traum war zuallererst eine »Vision« (vgl. oben tabrît mûši), ein Schauspiel.

7 Dennoch war nicht jede Vision, selbst wenn sie unnatürlich war, ein Traum. Beispielweise unterschied man sehr wohl die Fälle, in denen man von Verstorbenen träumte, von denen, in denen man sie bei vollem Tageslicht erscheinen sah. ${ }^{5}$ Ganz klar 
war man sich wie andernorts vollkommen bewusst, dass die Gegenstände dieser Traumspektakel eine besondere Konsistenz und Gestaltung aufwiesen, die sie sowohl von denen unterschieden, die man um sich herum sah wie von den »übernatürlichen« Erscheinungen. Das ist sicherlich auch der Grund dafür, dass man sich einen

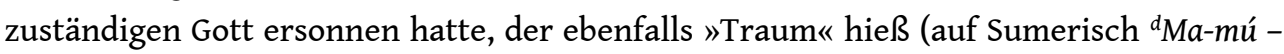
vgl. oben), vor allem aber auf Akkadisch "Leichter Hauch" ('Ziqîqu), eine bekannte Bezeichnung, die hier und da häufig verwendet wird, um die lose Konsistenz der "Gespenster" der Verstorbenen zu bezeichnen, diese Art doppelter - nicht greifbarer und entkörperlichter - Verschwommenheit, auf die sie sich nach ihrem Tod reduzieren. Daher musste die Traumwelt in Mesopotamien als ein in gewisser Weise luftiges Universum wahrgenommen werden - verschwommen, nicht greifbar und sozusagen immateriell.

Ein weiterer Unterschied zu der uns im Wachzustand umgebenden dreidimensionalen Welt besteht darin, dass der Traum für vollkommen unbekannte, ja unvorstellbare Erfahrungen im bewussten Leben Tür und Tor öffnete. Und das nicht nur dadurch, dass er jeglicher Logik entbehrte (man träumte, dass man »ohne Kopf« sei oder "gerade dabei war, seinen Penis zu verspeisen « ... vgl. unten), sondern auch aufgrund anderer ungewöhnlicher Umstände wie etwa der extremen Beweglichkeit, die den Autor eines Briefes dazu brachte, seinem entfernten Briefpartner gegenüber zu äußern: Alles, was $\mathrm{Du}$ dort unten tust, tragen mir meine Träume $z u .{ }^{6}$ Doch so verschieden Traum- und Alltagsleben auch sein mochten, sie hatten nicht weniger ein und dasselbe Individuum zum Gegenstand, das von dem einen Stadium in das andere überging, und dies manchmal in ein und demselben Moment (etwa wenn es nach einem Albtraum aus dem Schlaf hochfuhr - negeltû; tebû), und sich darüber vollkommen klar wurde, dass es die Welt der Träume verlassen hatte, um wieder auf die Erde zurückzukehren.

Kurzum, das Traumleben war nur eine Möglichkeit, eine Modalität, ein Moment des Lebens an sich; eine geheimnisvolle, unter besonderen (und scheinbar nie eingehender analysierten) Umständen sich vollziehende Fortsetzung des bewussten Daseins, wobei der Unterschied durchaus wahrgenommen wurde, ohne dass jedoch Traumwelt und bewusstes Leben im Gegensatz zueinander standen. Und gerade darum hatte sich dieses Traumleben ebenso sehr wie das bewusste Leben so gut in das Weissagungssystem einfügen können.

10 An erster Stelle in der inspirierten Weissagung steht die inspirierte Traumdeutung. Wie gezeigt, gründet sie auf dem Glauben, dass die Götter frei sind, das, was sie als einzige wissen, direkt wem auch immer sie wollen mitzuteilen, und dass sie nach Belieben dafür den Traum als Rahmen und als Vehikel wählen können. In der Sintfluterzählung ${ }^{7}$ will der Gott Éa die Menschen vor der Vernichtung schützen und hierfür mit folgenden Worten dem babylonischen Noah die bevorstehende Katastrophe offenbaren. »Ich habe den Atrahasis einen Traum sehen lassen, und so hat er das Geheimnis der Götter erfahren.«

11 Einen solchen Glauben an göttliche Traumoffenbarungen gibt es im antiken Mesopotamien schon von alters her. Das früheste Beispiel findet sich auf der bekannten "Geierstele«, deren Verfasser - der im Kampf gegen Umma befindliche König von Lagaš, E-ana-tum I. (um 2450 v. Chr.) - erzählt, wie ihm der Gott Ningirsu im Schlaf erschienen ist, um ihm in folgenden Worten Gewissheit über den Ausgang des Krieges zu verschaffen: „Nicht einmal Kiš unterstützt Umma! Der Gott Utu ergreift Partei für Dich! ... $\aleph^{8}$ Und wir wissen von vergleichbaren Äußerungen, angefangen mit dem 
altbabylonischen König Ammi-ditana, der im Traum aufgefordert wurde, den Göttern eine Statue von sich darzubieten', über die neuassyrischen Zeiten des Assurbanipal (um 630) bis zum letzten neubabylonischen Herrscher Nabonid (um 550). ${ }^{10}$ Solche privaten Traumoffenbarungen sind auf jeden Fall außergewöhnlich, vielleicht auch, weil die erhaltenen Dokumente in erster Linie öffentliche Angelegenheiten betreffen. ${ }^{11}$

Selbst wenn ihre Botschaft sich an den Herrscher richtete, scheinen die Götter einfache Untertanen als Mittelspersonen vorgezogen $\mathrm{zu}$ haben. Es folgt ein ausführliches Beispiel, anhand dessen man sich leichter ein Bild vom Funktionieren des Systems machen kann. In einem um 1770 an den König von Mari, Zimri-Lim, gerichteten Brief erklärt der Statthalter einer Provinz des Nordens diesem:

»An eben jenem Tag, an dem ich meinem Herrn das vorliegende Täfelchen sende, ist ein Bewohner von Šakâ, ein gewisser Malik-Dagan, mit folgenden Worten zu mir gekommen: >Ich beabsichtigte im Traum, mit einem Begleiter von Saggaratum nach Mari zu gehen. In Terqa angekommen, betrat ich sogleich den Tempel von Dagan, um mich vor diesem niederzuwerfen. Während ich so da lag, richtete Dagan folgende Frage an mich: "Sind die Scheichs der Jaminiten und ihre Leute mit den Leuten von Zimri-Lim, die (hierher) kommen, $\mathrm{zu}$ einem friedlichen Übereinkommen gelangt?« Ich antwortete: »Sie sind $\mathrm{zu}$ keinem friedlichen Übereinkommen gelangt!« Und gerade als ich den Tempel verlassen wollte, sprach er erneut: »Warum bleiben dann die Leute von Zimri-Lim nicht ständig bei mir, um mir seine Sache in allen Einzelheiten auseinanderzusetzen? Hätten sie es getan, ich hätte Zimri-Lim schon lange die Scheichs der Jaminiten ausgeliefert. Ich gebe dir daher jetzt den Auftrag, Folgendes Zimri-Lim auszurichten: >Befiehl deinen Gesandten, mir Deine Sache in allen Einzelheiten auseinanderzusetzen! Dann werde ich die Scheichs der Jaminiten ergreifen und sie dir ausliefern.« " Das behauptet dieser Mann im Traum gesehen zu haben. Ich sende heute eine Nachricht an meinen Herrn, damit er darüber nachdenke. Und wenn mein Herr will, dann möge mein Herr seine Sache Dagan darlegen und zu diesem Behufe die Gesandten meines Herrn regelmäßig zu Dagan schicken. Der Mann, der mir das erzählt hat, muss Dagan ein Tier zum Opfer darbringen, weshalb ich ihn dir nicht geschickt habe. Weil dieser Mann andererseits (im Übrigen) vertrauenswürdig ist, habe ich nichts von seinem Kopfhaar genommen oder von seinem Gewandsaum ..." (Revue d'Assyrologie, XLII, 1948, S. 128 ff.). ${ }^{12}$

Der gesamte Mechanismus ist hier aufgedeckt: Eine Privatperson erfährt im Traum eine Offenbarung, die eine öffentliche Angelegenheit betrifft. Er berichtet sie sogleich einem Vorgesetzten, der sie eilig dem Herrscher überbringt, damit dieser daraus die Folgerungen zieht. Wir können nachvollziehen, dass er, der Regel entsprechend, wenn schon nicht die Wahrhaftigkeit der Erzählung - ein unmögliches Unterfangen -, so zumindest doch die Glaubwürdigkeit dessen, der sie erzählt, hätte kontrollieren müssen, und zwar, indem er ihn selbst dem König übergeben oder diesem zum Zweck einer möglichen Überprüfung, deren Verfahren wir nicht kennen, etwas Persönliches wie etwa eine Haarlocke oder eine Kleiderfranse geschickt hätte. ${ }^{13}$ Doch wird die Überprüfung bei dem für seine "Glaubwürdigkeit» bekannten Träumenden für überflüssig eingeschätzt. Anders gesagt, bieten solche Träume leicht Anlass für gewisse Schwindeleien, und blickt man hinter die Kulissen, so lassen sich gar »Pressure Groups « ausmachen, angefangen mit dem Klerus des fraglichen Tempels von Dagan.

Wenn andererseits in dem zitierten Beispiel die Botschaft des Gottes zumindest ihren Empfängern klar genug erscheint (wir wissen von Fällen, in denen der Traum wie die Botschaft mehrere Tage hintereinander wiederholt werden, damit die Dinge noch klarer werden ${ }^{14}$ ), so haben wir auch andere, in denen sie rätselhaft erscheint, ob sie nun ausgesprochen oder in mehr oder weniger bildhaften Gesten oder Situationen zum 
Ausdruck gebracht wurde. Im Folgenden geben wir eine beredte Kostprobe aus einem anderen an Zimri-Lim gerichteten Brief wieder, dieses Mal von einer Frau, die im Palast von Mari eine wichtige Verwaltungsfunktion eingenommen zu haben scheint.

"Seit der Wiedererrichtung des Hauses meines Vaters [= Zimri-Lim, um 1780 nach dem Ende der assyrischen Herrschaft] hatte ich wirklich keinen vergleichbaren Traum mehr gehabt ... In meinem Traum betrat ich den Tempel der Belet-ekallim, doch war die Statue Belet-ekallims nicht da! Selbst die normaler Weise davor stehenden Statuen (der anderen Götter) waren nicht da! Angesichts dieses Schauspiels musste ich lange weinen. Diesen Traum hatte ich im ersten Drittel der Nacht. Danach hatte ich einen anderen: Dada, der Priester Ischtar-bischras hielt sich an der Pforte des Tempels der Belet-ekallim auf, während eine feindliche Stimme unaufhörlich schrie: /Komm zurück! Dagan! Komm zurück! Dagan!« «15

Für diesen Fall bleibt nicht nur die Situation unklar, sondern sogar die Ausrufe der feindlichen Stimme sind doppeldeutig aufgrund des Umstands, dass »Komm zurück! Dagan!" auf Akkadisch Tura-Dagan lautet, was zugleich der Name mehrerer zeitgenössischer, für uns sowohl bekannter als auch unbekannter Persönlichkeiten war. Bei solchen Träumen konnte also eine Deutung erforderlich sein.

Das ist der Daseinsgrund für die šẩilu, was zwar wörtlich »Prüfer, Fragensteller, Forscher « bedeutet, tatsächlich aber waren sie Spezialisten für die Auslegung derartiger Träume. ${ }^{16}$ Über ihre Technik wissen wir nichts. Es gibt Fälle, in denen sie als eine Art Kontrolle auf »objektive" (s. unten) Daten der deduktiven Weissagung zurückgreifen mussten. Es scheint aber, dass ihr Berufsstand sich vor allem auf persönliche Gaben stützte, auf eine gewisse Veranlagung, Begabungen, Scharfsinn und eine besondere "Inspiriertheit" - wenn man will so etwas wie bei unseren Kartenlegerinnen. Offenbar ist dies der Grund dafür, dass dieser Beruf sehr häufig von Frauen ausgeübt wurde, mehr oder weniger verwandt mit den Pythien und Hexen ( šâiltu). Eine berühmte Passage des altbabylonischen Gilgamesch-Epos kann uns zumindest eine Vorstellung von dieser Auslegungsmethode geben. Es handelt sich um die Erzählung des ersten Traums des Helden. ${ }^{17}$ Bevor er auf seinen späteren Freund und Begleiter Enkidu trifft, bekommt Gilgamesch in einem ersten Traum eine Vorahnung. Diesen »erzählt« (pašáru: »darlegen»)er seiner Mutter, die ihn ihm sogleich deutet (dasselbe Verb pašáru, im Sinne von »auslegen«)

»Es erhob sich da Gilgamesch, um den Traum zu lösen,

indem er zu seiner Mutter sagt:

`, meine Mutter, der Traum, den ich sah im Verlaufe dieser Nacht:

Da erschienen mir Sterne des Himmels.

Wie Brocken des Anum fallen sie immer wieder auf mich hernieder.

Ich hob einen an, doch er war zu stark über mir.

Ich brachte ihn immer wieder zum Wanken,

doch gelingt's mir nicht, ihn zu entfernen.

Uruk, das Land, steht da (gebeugt) über ihn.

Über ihm ist das Land versammelt.

Es drängt sich die Menge um ihn herum.

Die jungen Männer kommen um seinetwillen zuhauf.

So, als sei er ein kleiner Säugling, küssen sie ihm die Füße.

Ich liebte ihn wie eine Gattin und liebkoste ihn.

Ich hob ihn hoch und warf ihn dann dir zu Füßen.

Du aber wirst ihn mit mir auf eine Stufe stellen!

Die Mutter Gilgameschs ist klug, sie ist weise,

über alles weiß sie Bescheid. Sie sagt zu ihrem Sohn, Wildkuh-Ninsunna ist klug, sie

ist weise,

über alles weiß sie Bescheid. Sie sagt zu Gilgamesch: 
`Es erschienen dir Sterne des Himmels.

Wie ein Brocken des Anum fiel er auf dich hernieder.

Du hobst ihn an, doch er war zu stark über dir.

Du brachtest ihn immer wieder zum Wanken,

doch gelingt's dir nicht, ihn zu entfernen. $^{18}$

Und tatsächlich wird der in der Steppe geborene und in der Wildnis aufgewachsene Enkidu, der ebenso stark ist wie Gilgamesch (er ist ein »Brocken«) und von den Göttern geschickt wurde (er ist »vom Himmel gefallen«), in Uruk eintreffen, »umdrängt von der Menge« und »bewundert von den jungen Männern«. Zunächst wird sich Gilgamesch noch mit ihm messen, will ihn »hochheben" und zur Strecke bringen, dann wird er Freundschaft mit ihm schließen und ihn zu seiner Mutter »führen«, damit sie diese Bruderschaft segne. Für die Auslegung dieses Traums ist die Mutter Gilgameschs in Wirklichkeit so vorgegangen, dass sie eine Art Eingebung mit einem Wissen um die Zukunft gemischt hat. Daran ist nichts von "technischer" Strenge, nichts ist mit dem vergleichbar, was wir noch von der deduktiven Traumdeutung hören werden. Die weiteren Träume Gilgameschs und Enkidus - es gibt mehrere davon, darunter manche, über die man offen gesagt lange brüten kann - werden, zumindest insoweit uns die relevanten Passagen des Epos überliefert sind, alle mehr oder weniger nach einem analogen, im Wesentlichen intuitiven Verfahren interpretiert. Es gab gar, so scheint es, einen Fall von »Klarsicht«: Enkidu nimmt im Traum tatsächlich an der Götterversammlung teil, auf der sein Tod beschlossen wird (hethitisches Fragment, das dem Anfang von Tafel VII entspricht). Eigentlich ein klarer Fall, aber man könnte sagen, dass Gilgamesch (Ninive-Version VII/II) zur Beruhigung seines angesichts dieses Traumes bestürzten Freundes versucht, ihm einzureden, zum Verständnis dieses Traums müsse man die Bedeutung umkehren, dann könne ein so schrecklicher Traum eigentlich nur beruhigend sein ...

Kommen wir nun zur deduktiven Traumdeutung. Wir werden sehen, dass sie - den übernatürlichen Charakter beider Quellen wie auch die Mittel gegen die Unannehmlichkeiten, die sie mit sich bringen, einmal beiseite gelassen - der inspirierten Traumdeutung diametral entgegengesetzt ist.

17 Erstens war sie für Jedermann von Belang. Es drehte sich hier nicht mehr um außergewöhnliche Träume und übernatürliche Botschaften, die ja doch vielleicht häufiger den Großen dieser Welt vorbehalten waren, sondern um gewöhnliche, alltägliche Träume des "Mannes auf der Straße«, die für Jedermann galten. Wer auch immer träumte und egal wovon, der war auch Adressat der darin enthaltenen Botschaft. Nur war das eine "schriftliche« und »kodierte" Botschaft; und um sie zu lesen, bedurfte man eines veritablen Technikers, eines in diese Schrift eingeweihten Spezialisten, eines bârû, wie man sagte, also eines "Überprüfers«, jemand, der sich die im Traum enthaltenen "Bildzeichen" "genau besah" und "untersuchte«, der sie entzifferte und dem ihn aufsuchenden Betroffenen übersetzte. Und wie um die geringe Bedeutung zu bekräftigten, die für diese Technik das Temperament, die natürliche Verfasstheit und die Inspiration spielten, wird sie (wie überdies das Schreiben überhaupt) nur ausnahmsweise von Frauen praktiziert.

Das älteste dieser »Traumbilder« ist aufgrund seiner sehr frühen Datierung (um $1700 \mathrm{v}$. Chr.), seines Kontexts und seiner Ausgestaltung von Interesse. Es findet sich in einer kleinen Sammlung von ca. 60 Orakeln, die aus der Physiognomie wie aus dem menschlichen Verhalten sowohl am Tag wie in der Nacht stammen - eine Zusammenstellung, die betont, wie sehr das Traumleben alles in allem nur als eine 
besondere Ausdrucksform des Lebens angesehen wurde. Den Traum, um den es geht, würden wir als Albtraum bezeichnen. Er wird uns in zwei antithetischen Sätzen präsentiert, die beide mit einer schematischen Darstellung des Traums in Hypothesenform beginnen und mit der lakonischen Mitteilung der sich daraus ableitenden Zukunft enden.

»Wenn ein Mensch, während er schläft,( träumt, dass) eine/die Stadt wiederholt auf ihn fällt, [im materiellen Sinne des Einstürzens von Häusern oder bildlich als Feindseligkeit der Stadtbewohner?]

und Laute von sich gibt, ohne dass ihn jemand hört -

(was den) Mann (angeht): die Schutzgeister Lamassu und Šêdu sind an seinen Körper gebunden.

Wenn ein Mann, während er schläft, (träumt, dass) eine/die Stadt wiederholt auf ihn fällt,

und er Laute von sich gibt und jemand hört ihn:

(dann) ist ein übler Šēdu an seinen Körper gebunden. $\ll^{19}$

Eine vergleichbare Darstellung findet sich für sämtliche Orakel, und das nicht nur auf dem zitierten Täfelchen, sondern in sämtlichen Weissagungssammlungen, die in dieser Zeit bereits zahlreich (etwa hundert) und vielfältig (vor allem Eingeweideschau; auch Physiognomie, die Weissagung anhand von neugeborenen Menschen und Tieren sowie von Missbildungen, ja gar Astrologie und andere Sektoren der deduktiven Weissagung) vorkommen. Folglich musste man in dem Land bereits seit geraumer Zeit die Gewohnheit haben, diese universelle deduktive Weissagung auszuarbeiten. Und es spricht vieles dafür, dass der oben erwähnte Albtraum nur einen kurzen Auszug aus irgendeinem bereits entwickelten "Traumdeutungshandbuch" darstellte, das später noch erweitert wurde.

Unsere Quellenlage ist jedoch zu dünn und lückenhaft, als dass wir in der Lage wären, seine spätere Geschichte weiterzuverfolgen. Wir verfügen nur über das, was wir als eine Etappe ansehen können: eine in Suse gefundene und wahrscheinlich auf die zweite Hälfte des zweiten Jahrtausends zu datierende, äußerst bruchstückhafte Sammlung von etwa hundert Orakelträumen; und das Endergebnis: das klassische Traumbuch über deduktive Traumdeutung, den kanonischen babylonischen großen »Traumschlüssel«, dessen wichtigste überlieferte Handschriften aus dem 7. Jahrhundert stammen, von dem wir aber auch Fragmente besitzen, die ebenfalls in die zweite Hälfte des zweiten Jahrtausends zurückreichen. Dieses - wie es die Gewohnheit war - nach seinem Incipit Ziqiqu, Ziqiqu ... bezeichnete Werk dürfte im ganzen elf Täfelchen umfasst haben. Wir werden auf das erste und die beiden letzten eingehen, die nicht unmittelbar weissagenden, sondern exorzistischen Inhalts sind. Das aus acht Täfelchen (II bis IX) von jeweils 400 oder 500 Orakeln bestehende Korpus des Werks dürfte ein sehr ansehnliches Ganzes von mindestens drei- oder viertausend Traumsituationen gebildet haben. Unglücklicher Weise ist uns nur ein lückenhafter kümmerlicher Rest von ca. einem Fünftel überliefert. Doch wenn solche verstreuten Überreste uns auch nicht erlauben, seine Gliederung zu rekonstruieren, so können wir uns zumindest eine grobe Vorstellung davon machen und die Prinzipien wie die Methoden erkennen. ${ }^{20}$

Diese Abhandlung setzte sich in ihrer formalen Darstellung aus exakt dem oben erwähnten "Albtraum« nachgezeichneten Elementen zusammen. Ein jedes fing mit einer Hypothese (das, was die Grammatiker eine "Protasis« nennen) an, um das Thema des als Omen zugrunde gelegten Traumes hervorzuheben, und endete mit einer "Apodosis«, um daraus das entsprechende Orakel zu entwickeln. Wie in allen anderen Abhandlungen und Sammlungen von Weissagungen waren diese Omina sorgfältig nach 
ihrem Hauptgegenstand klassifiziert. So ergeben sich eine Art von Kapiteln, die etwa Reiseträumen (Tafel I) gewidmet sind, oder Träumen, in denen es um den Genuss von Lebensmitteln oder Getränken geht (A), um die Herstellung und Fabrikation vielfältiger Waren (III/I ...), um Gegenstände, die der Träumende erhält oder sich nimmt (B) und eine ganze Menge weiterer Tätigkeiten: Beispielsweise gibt es einen langen Absatz über das Ausscheiden von Urin (VII, r: 1 bis Ende). Hier nun zwei genügend reichhaltige Auszüge aus dem »Traumschlüssel«:

\begin{tabular}{|l|l|}
\hline $\begin{array}{l}\text { Wenn ein Mensch träumt, dass er Hundefleisch } \\
\text { gegessen hat }\end{array}$ & Angriff; nicht erfüllter Wunsch \\
\hline $\begin{array}{l}\text { Wenn ... Dachsfleisch ... } \\
\text { (auf akkadisch:»Mörtelhund«) }\end{array}$ & Angriff \\
\hline Wenn ... Gazellenfleisch ... & Hautausschlag (?) \\
\hline Wenn ... Büffelfleisch ... & Sein Leben währt länger \\
\hline Wenn ... Fuchsfleisch ... & Hautausschlag; für den Kranken ist das ein \\
\hline gutes Zeichen \\
\hline Wenn ... Affenfleisch ... & Er wird Erfolg haben \\
\hline $\begin{array}{l}\text { Wenn ... das Fleisch (irgendeines weithin bekannten } \\
\text { Tieres) isst }\end{array}$ & Glück \\
\hline \hline $\begin{array}{l}\text { Wenn ... das Fleisch ( irgendeines weithin } \\
\text { unbekannten Tieres) isst }\end{array}$ & Unglück \\
\hline Wenn ... Menschenfleisch isst & Jemand wird ihm all seinen Besitz \\
\hline Wenn ... das Fleisch eines Verstorbenen isst & (selbes Orakel)... \\
\hline Wenn ... Aas isst & Erd großen Reichtum ernten \\
\hline
\end{tabular}

und so weiter; danach isst er sich selbst; er isst das Fleisch seines Nächsten (»Gefährten«); dann von verschiedenen Teilen seines eigenen Körpers, die Hände, die Füße, den Penis ... Des Weiteren folgen Mengen von Gerichten, die eine eindrucksvolle „Speisekarte« ergeben; dann verschiedene Fleischsorten; allerlei Früchte und Gemüse; dann Tierfutter: von Horntieren und von wilden Tieren (einmal, nachdem »man es beschnuppert hat«); Stroh, Häcksel, Holz, Schilfrohr ...; Ziegelstein; roher Lehm; Erde; Leder; Exkremente ... (A. f II: 3'-r I: 27').

Und hier noch die fragmentarisch erhaltene Passage, in der das Urinieren behandelt wird (Quellenangabe oben):

$\begin{array}{lllll}\text { Wenn sein Urin, durch seinen Penis } & \text { wird er keine Kinder haben. }\end{array}$

gelenkt, eine Mauer überflutet: 


\begin{tabular}{|c|c|}
\hline $\begin{array}{l}\text { Wenn ... eine Mauer und die (angrenzende) } \\
\text { Straße überflutet: }\end{array}$ & wird er Kinder haben. \\
\hline Wenn ... mehrere Straßen überflutet: & $\begin{array}{l}\text { wird er seines Hab und Guts beraubt und es wird } \\
\text { unter seinen Mitbürgern verteilt }\end{array}$ \\
\hline $\begin{array}{l}\text { Wenn er, nachdem sein Urin geradewegs } \\
\text { aus seinem Penis entwichen ist, sich davor } \\
\text { niederwirft: }\end{array}$ & wird ein von ihm gezeugter Sohn König werden. ${ }^{21}$ \\
\hline $\begin{array}{l}\text { Wenn er }[. . .] \text { damit eine Mauer besprengt } \\
\text { und [...]: }\end{array}$ & wird er Kinder haben \\
\hline $\begin{array}{l}\text { Wenn er mit seinem Urin Schilfrohr } \\
\text { besprengt: }\end{array}$ & wird er Kinder haben [...; Lücke]... \\
\hline $\begin{array}{l}\text { Wenn er sich mit seinem Urin die Hände } \\
\text { wäscht: }\end{array}$ & $\begin{array}{l}\text { werden mehrere das Hab und Gut seiner Kinder } \\
\text { verschlingen (?). }\end{array}$ \\
\hline $\begin{array}{l}\text { Wenn er sich abtrocknet, nachdem er in } \\
\text { seinem Urin ein Bad genommen hat: }\end{array}$ & Ištar-Hand (genannte Krankheit). \\
\hline $\begin{array}{l}\text { Wenn er seinen Urin bis zum Himmel } \\
\text { spritzt: }\end{array}$ & $\begin{array}{l}\text { wird der von ihm gezeugte Sohn zwar eine } \\
\text { hochgestellte Persönlichkeit, sein eigenes Leben wird } \\
\text { jedoch verkürzt. }\end{array}$ \\
\hline Wenn er in einen Wasserlauf uriniert: & wird seine Ernte <überhaupt nicht $>$ reichlich ausfallen. \\
\hline Wenn er in einen Brunnen uriniert: & wird er sein Hab und Gut verlieren. \\
\hline Wenn er in ein bewässertes Feld uriniert: & wird der Regengott seine Felder überfluten. \\
\hline Wenn er auf einen Gott uriniert: & $\begin{array}{l}\text { [wird er nichts von dem], was er verloren hat, } \\
\text { [wieder bekommen]. }\end{array}$ \\
\hline
\end{tabular}

Nach einer ganzen Zahl von Annahmen steht am Ende des Täfelchens zu lesen:

\begin{tabular}{|l|l|}
\hline Wenn er seinen Urin im Sitzen laufen lässt: & Bekümmernis. \\
\hline Wenn er sich selbst anuriniert: & wird er alles vergessen, was er gesagt haben wird. \\
\hline Wenn er den Urin seiner Frau trinkt: & wird dieser Mann in großem Wohlstand leben. \\
\hline
\end{tabular}

23 Was uns an diesen Beispielen am meisten überrascht, ist die Systematik: Es werden, wenn schon nicht alle Hypothesen, was ja unmöglich wäre, so doch eine möglichst große Zahl abgehandelt und klassifiziert (nach einer für unser Denken mehr als einmal nicht zu durchschauenden Ordnung, deren Logik uns jedoch hier und da aufgeht: Hund $\rightarrow$ Dachs; Stroh $\rightarrow$ Häcksel $\rightarrow$ Ziegelstein $\rightarrow$ Lehm; Urin, der nur eine Mauer überschwemmt, dann auch die Straße, dann mehrere Straßen usw.); und das Ganze ergibt dann eine Art Kasuistik, die unter Rückgriff auf Analogiebildungen ermöglicht, 
diejenigen Schlüsse, die aus dem Behandelten gezogen sind, auf das nicht explizit Erwähnte auszuweiten. Das war im Übrigen im alten Mesopotamien das allgemein verbreitete Vorgehen sowohl in der Pädagogik wie bei der Darstellung wissenschaftlicher Inhalte.

Zum andern wird jede dieser gesammelten und katalogisierten Traumsituationen immer nur allein durch ihr Thema dargestellt, also das, was die Rolle eines »Bildzeichens" spielen und die Botschaft transportieren sollte; die konkreten Umstände des Traums, also das, was ihn zu etwas Besonderem und Persönlichen machte, werden beiseite gelassen - das ist übrigens auch der Grund, warum es uns nicht gelingt, anhand der Angaben des Traumbuchs einen "Zugang « zum vergangenen oder gegenwärtigen Privatleben der Träumenden zu finden. Es findet sich hier nie etwas »Erlebtes«. Die erotischen Träume ${ }^{22}$ sind beispielsweise nur durch ihren Gegenstand bestimmt, ohne dass etwas anderes erwähnt wird: Der Betroffene hat geträumt, dass er mit der Göttin der Liebe (Ištar), mit einem Gott, mit dem König, mit einem Würdenträger, mit einer Priesterin, mit der Königin, mit der Königstochter, mit der Gattin eines Anderen, mit dem Sohn eines Anderen, mit einer jungen Frau, mit einem jungen Mann, mit einem Knaben, mit seiner eigenen Tochter, mit seiner eigenen Schwester und gar mit einem Leichnam Verkehr gehabt hat. Doch niemals findet sich auch nur die geringste Anspielung an den Sitz im Leben ${ }^{23}$ des Traums, auf die Einzelheiten des Vorkommnisses, auf die dadurch ausgelösten Empfindungen und Gefühle usw. Offensichtlich zählt nur das zentrale Thema der Traumszene, weil sie allein gerade in ihrer Abstraktheit als bedeutungsvoll angesehen wird. Wie in der Bilderschrift sind das die Ähre, der Fuß, der Berg, die Frau als solche und nicht eine bestimmte Ähre, dieser Fuß, dieser Berg, jene Frau. Am Rande bemerkt, überzeugt uns gerade die Feststellung eines derartigen Bemühens um Abstraktion davon, dass wir hier nicht von der Suche nach dem Individuellen und Zufälligen sprechen, sondern nach dem Allgemeingültigen und Notwendigen - mit anderen Worten von »Wissenschaft«. ${ }^{24}$

Diese Feststellung muss auch auf die in solchen "Bildzeichen" entzifferten »Botschaften" ausgeweitet werden. Auch die Orakel stellen sich fast nur in verallgemeinerter Form und ohne den geringsten konkreten und individuellen Zug dar: »Unerfüllter Wunsch«, »Angriff«, »Hautausschlag«, »längeres« oder »kürzeres Leben«, »Erfolg«, »(Große) Reichtümer«, »Glück« oder »Unglück«, »er wird Kinder bekommen«, »einer seiner Söhne wird eine angesehene Person« oder "König" usw. Mit der Zeit nähern sich die Sammlungen von Weissagungen in der Formulierung ihrer »Apodosis" tendenziell zwar so nah wie möglich einem »Ja/Nein«, anders gesagt, einer "günstigen/ ungünstigen Antwort auf die gestellte Frage« an, wie man sie im Wesentlichen von den Wahrsagern erwartete. Ursprünglich scheinen sie jedoch überwiegend aus dem Alltagsleben entnommenen und entsprechend der eigentümlichen (s. oben) Sicht der Ereignisabfolge aufgezeichneten, auf den besonderen Fall bezogenen und auf Einzelheiten eingehenden Orakel bestanden $\mathrm{zu}$ haben. Und nicht wenige dieser "Fragmente des normalen Lebens" haben sich noch lange erhalten. Beispielsweise findet man in einer neobabylonischen Abhandlung über Eingeweideschau aus der Mitte des ersten Jahrtausends vor Christus noch das folgende veritable "Sittengemälde«: »Wenn (die Oberfläche der Leber so oder so aussieht), wird diese Frau, schwanger durch das Tun eines Anderen, unablässig die Göttin Ištar mit folgenden Worten anflehen: 'Wenn ich doch nur machen könnte, dass mein Kind wie mein Mann aussieht!«« ${ }^{25}$ In allem, was wir vom Traumbuch kennen, scheint man solche realistischen 
und aus dem Leben gegriffenen Visionen der Zukunft weggelassen zu haben zugunsten von Verallgemeinerungen, die unterm Strich auf ein »Ja« oder "Nein« hinauslaufen.

Dass die "vorausgesagte« Zukunft im genannten Traumbuch häufiger als etwas von der Situation des Träumenden Abhängiges dargestellt wird - analog zur Schrift, bei der die genaue Bedeutung eines Bildzeichens abhängig vom Kontext ist (»Land« oder »Ausland « für das Zeichen »Bergsilhouette«; »laufen«, »stehen « oder »mitnehmen« für den $\vDash \mathrm{Fu} \ll$ «) -, sollte man vielleicht als die Folge eines gewissen Widerstands gegen eine solche zunehmende Universalisierung der Orakel ansehen.

Wenn er träumt, dass er das Fleisch eines Fuchses isst: Hautausschlag; für den jedoch, der (schon) krank ist, ist dies ein gutes Vorzeichen. (A f. II: 7)

Wenn ... dass er ein Siegelzeichen erhalten hat [...]: wenn es sich um einen angesehenen Mann handelt, wird er in Armut verfallen; wenn es sich um einen Armen handelt, wird er reich werden. (B f. I: 25)

Wenn er Flügel hat, hin und her fliegt und landet: sein Baugrund/Untergrund/ Einkommen ist nicht solide/beständig ${ }^{26}$, (und) wenn er arm ist, wird ihn sein Unglück verlassen; wenn er reich ist, wird ihn sein Glück verlassen (C r. II: 21)

Und fast unmittelbar darauf (25):

Wenn ... dass er Flügel hat und sich emporschwingt und davonfliegt: wenn er arm ist, wird ihn sein Unglück verlassen; wenn er reich ist, wird ihn sein Glück verlassen; wenn er im Gefängnis sitzt, wird er entlassen und seine Freiheit wiederbekommen; wenn er krank ist, wird er genesen.

Vielleicht sollte man Analoges voraussetzen, wenn es (was nicht ungewöhnlich ist) vorkommt, dass ein und derselbe Traum in derselben Hypothese mehrere unterschiedliche Zukunftsperspektiven offen lässt, die in der Folge als Varianten dargestellt werden:

Wenn ... dass er Alraunwurzeln isst [auf Akkadisch: »Schicksalspflanze «]: er wird

(vor Gericht) über seinen Gegner siegen; Einberufung( in den Militärdienst);

schreckliche Neuigkeit. (A r. IIa: 8ss)

Von den vier hier vorgesehenen "Schlüssen" enthalten mindestens zwei schlechte Vorhersagen, eine ist günstig! Eine solche Vielfalt, ja Widersprüchlichkeit, birgt möglicherweise die Erinnerung an die "experimentellen« Ursprünge der deduktiven Weissagung: Das gleiche ungewöhnliche Vorkommnis könnte, je nach Fall, sehr wohl verschiedene Situation nach sich ziehen. Es lassen sich dahinter auch verschiedene "Interpretationsschulen« vermuten.

Doch wie im vorangegangenen Fall könnten diese Varianten auch das Wissen um eine gewisse Doppeldeutigkeit und vielfältige Auslegbarkeit der Bildzeichen in der Weissagung wie im Schriftgebrauch verraten.

Bleibt noch das Problem des Lektüre-»Kodes" dieser "Traumbildzeichen«. Was bestimmte ihre Bedeutung und autorisierte die bârû, die sie in Augenschein nahmen, sie auf ausreichend eindeutige und gesicherte Art und Weise zu »entziffern « und ohne jegliche Willkür oder Phantasie vom Vorzeichen zum Orakel - von der Protasis zur Apodosis zu schreiten? Eingedenk der Jahrtausende wie der grundlegenden ideologischen Unterschiede, die uns von den damaligen Nutzern der babylonischen Traumdeutung trennen, müssen wir darauf gefasst sein, dass es uns weder leicht noch überhaupt häufig möglich sein wird, die Logik des Zusammenhangs von Zeichen und Bezeichnetem zu verstehen, was sowohl für die gewöhnliche als auch für die in den Weissagungen verwendete Bilderschrift gilt.

In den Wahrsage- und Traumdeutungsbotschaften scheint diese semantische Verbindung häufig auf einer realen, imaginären, analogiebasierten oder rein 
konventionellen Beziehung zu fußen, die aus dem einen das Zeichen oder Symbol des anderen macht. Für den, der träumt, dass er die Früchte des Rebstocks verzehrt, ist es selbstverständlich, dass man ihm entweder Freude oder eben - als häufige unangenehme Folge des Genusses dieser Leckerei - eine "Verdauungsstörung« verheißt (A f. IV: 10). Im Traum wie im alltäglichen Leben wahrt das Trinken von Wasser die Gesundheit und »verlängert das Leben« (B r. IIa: 14), übermäßiger Genuss von Wein "verkürzt es« (Ebd. I: 9 und IIa: 16), während zu viel Bier bewirkt, dass man den Kopf verliert. Aus diesem Grund "weiß» der, der träumt, dass man ihm Bier reicht, "nicht mehr, was er sagt (Ebd. I: 10 und IIa: 15). Dieser pathologische Verlust des Bewusstseins findet sich auch auf einem anderen Gebiet: Auf sich selbst zu urinieren impliziert im normalen Leben einen Mangel an Selbstkontrolle, wenn nicht gar den Verfall der geistigen Kräfte. Es ist daher nicht erstaunlich, dass ein ähnlicher Vorfall im Traum den gleichen Verfall ankündigt (VII, in: 2's). In sämtlichen Fällen wurden die Eigenheiten und Auswirkungen der Dinge des bewussten Universums einfach in die Traumwelt transferiert. Moderne »Traumdeutungsbücher « verfahren diesbezüglich kaum anders, wenn sie uns beispielsweise bezüglich des im Traum ansichtigen Meeres erklären: »Ist es schwarz und aufgewühlt, dann ist dies ein schlechtes Vorzeichen; wenn wir mit einem schönen Schiff darauf herumfahren, steht das für ein Schicksal, in dem wir alles im Griff und unter Kontrolle haben, für Sieg über die finsteren Kräfte des Unbewussten « ...

Die Dinge sind zumindest für uns manchmal ein wenig komplizierter, und um den Übergang von der Protasis zur Apodosis zu erläutern, müssen wir auf eine eingeborene Sichtweise oder Gewohnheit, die uns fremd geworden ist, zurückgreifen. Warum sieht sich derjenige, der träumt, dass er, "nachdem er in einen Flusslauf getaucht ist, wieder auftaucht«, vergewissert, »dass er (vor Gericht) über seinen Gegner siegt« (C r. II: 44)? Weil sein Traum auf das damals im Land geläufige Ordalienverfahren anspielt: Wenn das Aktenmaterial dem Richter keine Entscheidung beim Urteil erlaubte, verwies er die beteiligten Parteien auf das Urteil der Götter, in diesem Falle also auf den Fluss-als-vonihnen-beauftragte-übernatürliche-Macht. Wenn der Angeklagte wie ein Stein darin unterging, bewies der Fluss damit seine Schuld. Wenn er ihn wieder »nach oben brachte«, erklärte er ihn für unschuldig und zum Gewinner des Prozesses. ${ }^{27}$ Genauso musste derjenige, der träumte, dass er mit seiner (noch unter seiner Obhut stehenden) Tochter (also vor ihrer Eheschließung) Verkehr hatte, mit einem »Verlust seiner Geldeingänge rechnen« (Susa, III: 9): Das lag daran, dass der Betreffende die erhoffte Ausgleichszahlung - das, was man terhatu nannte -, die gewöhnlich die Schwiegereltern der Familie der Braut auszahlten, nicht in der erhofften Höhe erhielt, weil die Eheschließung aufgrund der Entjungferung der jungen Frau schwieriger, wenn nicht gar unmöglich geworden war.

Bei dieser Verlegung der Dinge des bewussten Lebens in die Traumwelt kam es aus Gründen, die wir nicht kennen, zu einer Bedeutungsumkehrung. In dem weiter oben erwähnten Albtraum hatte derjenige, der im Traum nicht gerettet wurde, im wirklichen Leben Glück und umgekehrt. „Wer träumt, dass er auf der Erde sitzt: der wird Ehren empfangen " (III, f. II: 6); und Oppenheim (1956), S. 266a, hat die Beobachtung gemacht, dass die geträumten Verletzungen der Verbote und Tabus, völlig im Gegensatz zum wirklichen Leben, regelmäßig glückliche Ergebnisse nach sich ziehen. Wer im Traum seine eigenen Ausscheidungen isst, dem wird »eine Zunahme seines Hab 
und Gutes« versprochen (A r. I: 23), und "großer Wohlstand« wird dem verheißen, der unter denselben Umständen den Urin seiner Frau trinkt (VII r. II: Ende).

Bei der Entzifferung der Weissagungen spielt auch die Symbolik eine herausgehobene Rolle. Auch hier wissen es unsere Traumbücher wieder bestens (»Die Straße im Traum symbolisiert das Geschick, unseren "Lebensweg" ... "Der Berg steht für eine unüberwindliche Schwierigkeit ...«). Und wie wir schon gesehen haben, verspricht das Urinieren aufgrund der Gleichsetzung von Urin und Sperma beinahe überall "Kindersegen«. Genauso hat derjenige, der „träumt, wie er gerade seinen Penis vertilgt«d.h. ihn beseitigt -, mit dem Tod eines seiner Kinder zu rechnen (A f. IIb: 30). Die Semantik des Penis ist jedoch nicht eindeutig (was im Übrigen auch beim Schreiben anderer Bildzeichen der Fall ist). Er darf nicht nur als ein Zeugungswerkzeug, sondern muss auch als Zeichen männlicher Potenz gesehen werden. So wird etwa derjenige, der ihn sich "ganz lang" erträumt, "keinen Rivalen haben" (Susa, III: 18). Ein weiteres interessantes Symbol ist das Siegel. Als übliches Zeichen und Substitut für die Person verkörpert es regelmäßig auch jenes andere Kennzeichen, die Verlängerung eines jeden von uns, nämlich die Nachkommenschaft. Wenn es im Traum jemandem "gegeben« wird, vervielfachen sich die Apodosen. Der Träumende "wird als ältestes Kind einen Sohn haben « ( B f. I: 11); »er wird Söhne und Töchter bekommen« (13s; 17; 18; 20; usw.). Und wenn er träumt, dass man ihm sein Siegel stiehlt, "wird entweder sein Sohn oder seine Tochter ums Leben kommen« (Ebd.: 23) ...

In manchen Fällen besteht das, was die Verbindung zwischen der Weissagung und dem Orakel herstellt, aus einer einfachen Assonanz, dem, was wir ein Wortspiel nennen würden. So wird beispielsweise dem, der im Traum »einen Esel trifft" (auf Akkadisch imêru), das "Sehen (imirtu) von Kindern" versprochen (A r. IIIb: 6"). Diese eigenartige Wendung, meines Wissens nach ein Hapax, die schlicht besagen soll, dass er Kinder um sich sehen wird, dass er welche bekommt, wurde allem Anschein nach aufgrund des beinahen Gleichlauts von imêru gewählt. In einer teilweise verlorenen Protasis, in der zumindest das Hauptverb erhalten ist, sieht der Träumer, wie er [etwas] »isst« (akâlu), und die Apodosis verspricht ihm an erster Stelle, dass "er Leckereien essen (akâlu) wird" (wörtlich: süßes Brot - akalu; die allgemeine Bedeutung ist, dass er gut genährt = wohlhabend sein wird); dann, als Variante oder Folge dessen, „Verfinsterung" (etkulu) (IV f. I: 19s). Wer sich im Traum in das Land Laban (Libanon?) begibt, "wird für sich ein Haus bauen « (IX: Sm 2- +: 10's) - weil der Name dieses Landes das Verb labànu: „Ziegel herstellen « evoziert. Und der Begriff hat[tu], Schrecken, Panik, steht in der beschädigten Apodosis des Traums von einer Reise ins Land Hatti (zu den Hethitern) (IX, r. I: 7). Doch sind das alles unserem Dafürhalten nach keine einfachen "Wortspiele«. In diesem Land, in dem die Namen nicht als willkürliche und letztlich subjektive Epiphänomene verstanden wurden, sondern als objektiver Ausdruck des eigentlichen Wesens der Dinge, war jegliche phonetische Übereinstimmung etwas in hohem Maße Ernstes und Bedeutungsvolles. Zwei Realitäten, deren Namen in eins fielen, fanden sich ebenso eng miteinander verbunden wie ihre Bezeichnungen.

Eine weitere mögliche Technik der "Lektüre» von Traumbildzeichen kommt in der Deutung des Traumes einer Reise in das (unbekannte) Land Itrân zum Vorschein. Man verheißt dem Betreffenden, dass »er sich einer Strafe entledigen wird" (IX r. I:21). Dieser letztere Begriff wird im Akkadischen mit aran wiedergegeben; und der Name des betreffenden Landes schreibt sich mit einem ersten Zeichen IT, das sich auch als À lesen lässt: von It-ra-an (gesetzt den Fall, dass man es genau so buchstabieren muss) kommt 
man leicht zu À-ra-an, das gleichlautend mit aran ist. Man muss dabei wissen, dass in der Keilschrift die recht häufige Homophonie der Zeichen und die regelmäßig anzutreffende, gleichermaßen phonetische wie ideographische Mehrdeutigkeit sowohl im Sumerischen als auch im Akkadischen die Grundlage für eine in unseren Augen schwindelerregende »Logik« und eine ebensolche heuristische Methode abgegeben hat, die mit größter Ernsthaftigkeit häufig derartige Substitutionen vornehmen. ${ }^{28}$ Mit größter Wahrscheinlichkeit spielten zwischen den zahlreichen "Lesarten" von Weissagungsbildzeichen, die sich weder verstehen noch belegen lassen, derartige für unsere Augen schwer auszumachende Feinheiten eine Rolle, die zu postulieren mangels Informationen meist eine gewagte Angelegenheit darstellt. Aus diesem Grund bleibt ein Gutteil des "Lektürecodes« für diese Weissagungen für uns unverständlich. Im Folgenden ein zweifaches Orakel, in dem - und das ist selten - Protasis und Apodosis identisch sind und identisch ausgedrückt werden. Das eine dreht sich um einen "Fuchs", das andere um einen übernatürlichen »Glücksbringer", den man auf Akkadisch Lamassu nannte. Der, der im Traum einen Fuchs fängt, »fängt«, d.h. erhält einen Lamassu, in anderen Worten: er wird Glück haben. Doch wenn der Fuchs, nachdem er erst gefangen ist, wieder entwischt, entwischt der einmal »gefangene«Lamassu ebenso (B r. III: 9s). Es findet also eine völlige Assimilierung von Fuchs (auf Akkadisch šêlibu; auf Sumerisch $\mathrm{ka}_{5}-\mathrm{a}$ ) und Lamassu (auf Sumerisch Lamma; geschrieben AN.KAL). Doch auf der Basis welches vermittelnden Begriffs, welcher Beziehung zwischen ihnen, aufgrund welcher Symbolik, welches phonetischen oder graphischen, ja ikonographischen Spiels? Im Augenblick kann das niemand wissen. Und das ist nur allzu oft der Fall.

Wie es auch immer um diese Unsicherheiten bestellt sein mag, so steht doch fest, dass die Weissagungs- und Traumdeutungsapodosen keineswegs willkürlich waren und dass ein umfassendes, auf seine Weise logisches und von den Technikern - den bârû perfekt beherrschtes System von Äquivalenzen bestand, um sie aus den Protasen zu deduzieren und somit in den Vorkommnissen des Traumlebens das, was die Götter darin bezüglich der Zukunft hineingeschrieben hatten, lesen zu können.

Eine letzte Frage stellt sich noch: Um was für eine Zukunft handelt es sich dabei eigentlich? Ist sie absolut, unausweichlich oder nicht? Davon hängt nämlich die tiefe Bedeutung der Traumdeutung - und des Wahrsagens -ab. Wir müssen hier nochmals auf die Täfelchen I und X-XI des Korpus des Traumbuchs eingehen: Uns bleiben nur Bruchstücke, doch lassen sie sich teilweise mittels besser erhaltener Parallelstellen rekonstruieren. Was sieht man da? Sie enthalten im Wesentlichen das, was wir »Beschwörungen« nennen, oder, wenn man mir folgen will, besser: Gebete "sakramentaler « Natur. Das heißt, dass sie an die Götter gerichtet sind, um von ihnen die Beseitigung einer Bedrohung, eines Unglücks oder Übels zu erlangen, und aus einer Mischung sich wechselseitig verstärkender mündlicher sowie manueller Riten bestehen. Im Folgenden zwei sehr kurze Beispiele dafür:

»Wenn ein Mensch einen Traum mit schlimmen Vorzeichen geträumt hat, soll er, damit dessen schädliche Folgen ihn nicht treffen, sagen, bevor er seine Füße (am Morgen) auf den Boden setzt: >Dieser Traum, den ich hatte, ist ein gutes Vorzeichen, er ist ein gutes Vorzeichen, er ist ein ausgezeichnetes Vorzeichen im Namen Sins und Šamaš! ‘ Auf diese Weise wird er sich (anstelle des schlimmen) ein gutes Omen geben und das im Traum vorausgesagte böse Geschick wird ihn nicht treffen!« (Sm 1069 r. 3ss/ /KAR 252, I: 7 ss; Oppenheim (1956), S. 300b). Und:

" $[$ [...] halte fern von mir und zerstreue den Fluch (der mich bedroht)! Der Wind möge das mir im meinem Traum vorausgesagte Schlimme Geschick weit weg 
tragen! So lautet das Gebet zur Fernhaltung der schädlichen Folgen des betreffenden Traumes. Man begleite es mit folgendem manuellen Ritus: wenn der Betroffene einen solchen Traum hatte, bevor er am Morgen die Füße auf den Boden gesetzt hat, soll er (dort) ein Essigopfer darbringen (und das oben aufgeführte Gebet sprechen), und erst nachher soll er seine Füße auf den Boden setzen. Auf diese Weise wird das aus seinem Traum zu erwartende böse Geschick von ihm ferngehalten!« (K 13330/ /KAR 252, III: 47ss; Oppenheim (1956), S. 300b). deduktiven Traumdeutung legt nahe, dass es kaum nur um die darin niedergelegten Träume gehen kann, nämlich die, die schlechte Omen oder ein gegenteiliges Schicksal verheißen. Gerade ihr Vorhandensein belegt, dass man also gegen das schlimme Schicksal, wenn es - wie alles, was für den Lauf der Welt und ihrer Bewohner von Bedeutung ist - von den Göttern zwar mitgeteilt und vorher von ihnen beschlossen wird, dennoch mittels eines Rückgriffs auf die eigentlichen Urheber dieses Beschlusses und seiner "schriftlichen" Übermittlung vorgehen und es sogar aufheben lassen konnte. Und in Anbetracht dessen, dass die Art und Weise der Kommunikation - und nur dadurch lassen sich im Großen und Ganzen die deduktive und die inspirierte Traumdeutung voneinander unterscheiden - absolut sekundär hinsichtlich der Bedeutung des mitgeteilten Beschlusses ist, können wir bedenkenlos davon ausgehen, dass sich der gleiche Rückgriff auch für den Inhalt der »übernatürlichen« Träume praktizieren ließ. Das durch die deduktive wie die inspirierte Traumdeutung - und noch allgemeiner durch die deduktive wie die inspirierte Wahrsagekunst - offengelegte Zukünftige war also nicht die unbedingte Zukunft, das, was auf jeden Fall und unausweichlich eintraf, sondern eine bedingte Zukunft. Ich habe sie judikative Zukunft genannt, weil ihr Beschluss und dessen Folgen exakt von denen der Gerichtsurteile übernommen zu sein scheinen. Wenn der Richter über das Schicksal eines seiner Zuständigkeit Unterworfenen entscheiden muss, dann hat er vor sich eine Anzahl von Anhaltspunkten, anhand deren er diese Entscheidung fällt. Auf die gleiche Weise stützen sich die Götter - bezüglich deren im antiken Mesopotamien die gesamte Lehre aus einer Übertragung der auf Erden ausgeübten Macht besteht -, wenn sie über das Schicksal eines jeden Menschen entscheiden, auf die Angabe einer ganzen Anzahl von Umständen, und sobald eine solche Entscheidung getroffen ist, teilen sie diese entweder unmittelbar oder »in schriftlicher Form « und insbesondere - das ist ja unser Thema - über Träume mit. Ob diese Letzteren nun unmittelbare und »inspirierte« oder »schriftliche« und »zu deduzierende« Mitteilungen sind, ihr Inhalt ist alles in allem nur ein Urteilsspruch, der nicht angibt, was dem Betreffenden ganz sicher zustoßen wird, sondern was ihm für den Augenblick verheißen ist und auf was er sich gefasst machen muss. Und dieser Inhalt kann wie der eines jeden von Umständen abhängigen Urteilsspruchs Gegenstand eines Appells an die Milde des Richters sein.

Das ist nun der Grund dafür, dass man nicht nur die erwähnten »Exorzismen« der Täfelchen I und X-XI des Traumbuchs, sondern eine ganze Menge weiterer erarbeitet und verbreitet hat, für sämtliche Sektoren der Weissagung, für sämtliche Übermittlungsarten von göttlichen Entscheidungen bezüglich der Zukunft. Man nannte sie namburbû, Verfahren zur "Auflösung" des von den Orakeln verheißenen üblen Geschicks ${ }^{29}$, und sie verleihen der mesopotamischen Wahrsagekunst erst ihre wahre Bedeutung. Sie hatte keineswegs etwas Unausweichliches an sich und nahm auch überhaupt nicht irgendwie geartete metaphysische, unveränderliche Mächte, für alle Ewigkeit erlassene und sich unausweichlich vollziehende Entscheidungen in Anspruch. 
Solche Götter sind der mesopotamischen Theologie unbekannt. Für sie bestand die Rolle der Vertreter der übernatürlichen Welt darin, die Welt zu verwalten. Auf einer höheren Ebene, mit einer unendlich größeren Macht und Intelligenz sowie obendrein Unsterblichkeit ausgestattet, fungierten sie wie die Könige auf Erden. Sie regierten und regelten die Rolle und das Geschick ihrer Untertanen. Doch wie die Entscheidungen der Könige waren die ihren stets an Umstände gebunden und durchaus veränderbar, vorausgesetzt, man stellte sich geschickt an. Das eine waren die Entscheidungen, die sie direkt oder "in schriftlicher Form" durch Offenbarungen oder Traumvorzeichen bekannt gaben; etwas anderes waren die Entscheidungen, die die gebührend sachverständigen Betroffenen mittels Gebeten und passender Riten rückgängig machen konnten.

Daher werden die Wahrsagekunst im Allgemeinen und die Traumdeutung im Besonderen im antiken Mesopotamien nur verständlich, wenn sie wieder in das Denksystem der Bewohner dieser alten Länder, in deren umfassende Sicht vom Universum eingefügt werden.

\section{BIBLIOGRAPHIE}

André-Leickman, B. / Ziegler, C. (Hg.) (1982): Naissance de l'écriture. Cunéiformes et hiéroglyphes, Paris.

Annuaire 1973-1974 de l'École pratique des Hautes Études, IVème Section, Paris 1974.

Archives royales de Mari, X (1978): Correspondance feminine, Paris.

Archives royales de Mari, XIII (1964): Textes divers, Paris.

Beyerlin, W. (Hg.) (1985): Religionsgeschichtliches Textbuch zum Alten Testament, 2. Aufl., Göttingen.

Borger, R. (1988): Assyrisch-Babylonische Zeichenliste, 4. Aufl., Kevelaer.

Bottéro, J. (1977): »Les noms de Marduk, l'écriture et la >logique en Mésopotamie ancienne«, in: De Jong Ellis, M. (Hg.): Essays on the Ancient Near East in Memory of J. J. Finkelstein, Memoirs of the Connecticut Academy of Arts and Sciences 19, Hamden, Conn., S. 5-28.

Bottéro, J. (1981): »L'ordalie en Mésopotamie ancienne«, Annali della Scuola Normale Superiore di Pisa, Classe di Lettere e Filosofia, ser. III, vol. XI, Pisa, S. 1005-1067.

Bottéro, J. (1982): „De l'aide-mémoire à l'écriture«, in: Christin, A. M. (Hg.): Ecritures: systèmes idéographiques et pratiques expressives: Actes du Colloque International de l'Université de Paris VII, 22, 23 et 24 avril 1980, Paris, S. 13-38.

Bottéro, J. (1973): »Divination et esprit scientifique«, Sciences et Avenir, 313, S. 284-289.

Clay, A. T. (1923): Epics, Hymns, Omens and other Texts, BRM IV, New Haven.

Dietrich, M. et al. (1986): Texte aus der Umwelt des Alten Testaments, Bd. 2., Religiöse Texte, Lfg. 1., Deutungen der Zukunft in Briefen, Orakeln und Omina, Gütersloh. 
Gadd, C. J. (Hg.) (1925): Cuneiform Texts from Babylonian tablets in the British Museum, XXXVIII, London.

Falkenstein, A. (1966): »'Wahrsagung« in der sumerischen Überlieferung«, in: Wendel, F. (Hg.): La divination en Mésopotamie ancienne et dans les régions voisines. XIV Rencontre assyrologique internationale (Strasburg 2-6 juillet 1965), Paris, S. 45-68.

Finet, A. (1969): "Les symboles du cheveu, du bord de vêtement et de l'ongle en Mésopotamie«, Annales du Centre des anciennes religions, 3, Brüssel, S. 101-130.

Harper, R. F. (1914): Assyrian and Babylonian Letters, Chicago.

Herodot (1824): Die Geschichten des Herodotus, übers. von Friedrich Lange, Breslau.

Köcher, F. / Oppenheim, A. L. (1957/58): »The Old Babylonian Omen Text VAT 7525«, Archiv für Orientforschung, 18, S. 62-67.

Lambert, W.G. (1975): Babylonian Wisdom Literature, 3. Aufl., Oxford.

Langdon, S. (1912): Die neubabylonischen Koenigsinschriften, Leipzig.

Oppenheim, A. L. (1956): The Interpretation of Dreams in the Ancient Near East, Transactions of the American Philosophical Society, N.S. 46/3.

Oppenheim, A. L. (1969): »New Fragments of the Assyrian Dream-Book«, Iraq, XXXI, S. 153-165.

Maul, S. M. (2005): Das Gilgamesch-Epos, neu übersetzt und kommentiert von Stefan M. Maul, München.

Parpola, S. (Hg.) (1993): Letters from Assyrian Scholars, Helsinki.

Scheil, V. (1913): Mémoires de la Délégation en Perse, Bd. XIV. Textes élamites-sémitiques. Paris.

Sollberger, E. /Kupper, J.-R. (1971): Inscriptions royales sumériennes et accadiennes, Paris.

Streck, M. (1975): Assurbanipal und die letzten assyrischen Könige bis zum Untergange Niniveh's, II, Fotomechanischer Neudruck der Originalausgabe 1916, Leipzig.

Vernant, J.-P. et al. (1974): Divination et rationalité, Paris.

Walters, S. D. (1970): Water For Larsa. An Old Babylonian Archive dealing with Irrigation, (Yale Near Eastern Researches 4), New Haven / London.

Zgoll, A. (2006): Traum und Welterleben im antiken Mesopotamien. Traumtheorie und Traumpraxis im 3.-1. Jahrtausend v. Chr. als Horizont einer Kulturgeschichte des Träumens, Münster.

\section{NOTES}

1. Vgl. insbesondere Vernant et al. (1974), S. 70-197.

2. Dazu Bottéro (1982), S. 13-37.

3. Vgl. André-Leickman / Ziegler (1982).

4. Falkenstein (1966), S. 56 und Fn. 3 ff.

5. Während im Traumbuch die Toten im Traum erblickt werden (so Oppenheim [1956], S. 327 f., S. 66 und $72 \mathrm{ff}$.), so handelt es sich in dem Buch der "Zufälligkeiten des täglichen Lebens« (šumma âlu) offenkundig um Erscheinungen von Gespenstern (eŢemmu) oder »Toten als ob sie noch am Leben wären« (mitu kîma balţi): Man sieht sie, sie machen einem Angst, sie stoßen Schreie aus, halten sich am Bettende fest, kommen und gehen wieder, usw. Vgl. Gadd (1925), Tafel 25 f, 23 ff. und Tafel $30 \mathrm{f} ., 1 \mathrm{ff}$. 
6. Textes cunéiformes du Louvre I, $n^{\circ} 53$, S. 83.

7. Gilgameš ninivite, XI, 187 [= die heute oft als Standardversion bezeichnete Fassung aus der Bibliothek des Aššurbanipal in Ninive, die heute im British Museum aufbewahrt wird - A.d.Ü.].

8. VII, 17 ff; Sollberger / Kupper (1971), S. 49.

9. Reallexikon der Assyriologie, II, S. 187b: 223; um 1670.

10. Vgl. beispielsweise S. Langdon, Die neubabylonischen Königsinschriften, S. 218 ff; I: $16 \mathrm{ff.}$

11. Archives royales de Mari, $\mathrm{X}, 100$; Walters (1970), S. 93, Nr. 69: 4-10; Harper (1914), Nr. $1021=$ Parpola (1993), Nr. 294: 13 ff.

12. [Nach der französischen Vorlage übersetzt; eine deutsche, allerdings abweichende Übersetzung findet sich in: Beyerlin, W. (Hg.): Religionsgeschichtliches Textbuch zum Alten Testament, Göttingen $1985^{2}$, S. 149 f. Im Netz unter: http://books.google.de/books? id=FU2DMkLMdSIC\&pg=PA150\&dq=B\%C3\%A9let-

ekallim\&hl=de\&ei=uGwJToD5K4fRsgavu5TRDg\&sa=X\&oi=book_result\&ct=result\&resnum=2\&ved=0CDAQ6AEwAQ\#v=onepage\&q\&f=false 13. Finet (1969).

14. Vgl. Archives royales de Mari, XIII, Nr. 112: $\mathrm{r}$.

15. Archives royales de Mari, $\mathrm{X}, \mathrm{Nr} .50: 3 \mathrm{ff}$. [Zu einer abweichenden deutschen Übersetzung siehe Hinweis in Fußnote 12 - A. d.Ü.]

16. Vgl. Lambert (1975), S. 128 ff.: 54.

17. Dieser Held hat mehr Träume als andere, sein Freund Enkidu ebenso. Das Gilgamesch-Epos ist eine wahre Fundgrube für diejenigen, die mehr über die inspirierte Traumdeutung wissen wollen.

18. Gilgamesh Pennsylv.: 1-23. [= die zweite Tafel der überlieferten altbabylonischen Fassung, die heute in Philadelphia aufbewahrt wird (Pennsylvania tablets). Die deutsche Übersetzung fußt hier nicht auf der davon stark abweichenden französischen Übersetzung, sondern wird zitiert nach: Das Gilgamesch-Epos, neu übersetzt und kommentiert von Stefan M. Maul, München: Beck, 2005, hier: S. 54 f; im Internet auch unter http://books.google.de/books? $\mathrm{id}=$ zvhs04rMNmoC\&printsec=frontcover\&source=gbs_ge_summary_r\&cad=0\#v=onepage\&q\&f=false - A.d.Ü.]

19. Köcher, F. / Oppenheim, A. L. (1957/58), S. 67, III: 37s. Übersetzung nach Zgoll (2006), S. 444.

20. Vgl. Oppenheim (1956), wo die Abhandlung nach dem gegenwärtigen Stand abgedruckt und untersucht wird. Verwiesen sei auch auf Oppenheim (1969), S. 153 ff. Für den Text von Susa vgl. Scheil (1913), S. 49 ff. und Tafel VI. Die römischen Ziffern (Tafel I usw.) und die Großbuchstaben, mit denen die hier angeführten Zitate versehen sind, beziehen sich auf die Anordnung von Oppenheim (1956).

21. Es sei hier auf die von Herodot (I, $107 \mathrm{ff}$ ) erzählte Geschichte des Astyages hingewiesen: der seine Tochter im Traum sieht, "wie so viel Wasser von ihr ging, daß seine ganze Stadt davon erfüllt und ganz Asien überschwemmt ward. [...]. Denn es hatten ihm die Traumdeuter unter den Magern geweissaget, seiner Tochter Sohn würde König werden an seiner Statt...«.

22. In Oppenheim (1956), S. 334, X und 3 ff.; und für den Wert Tुehû »sich jemandem annähern«, "mit ihm/ihr schlafen«, des verwendeten Sumerogramms UM/DUB, das Oppenheim nicht übersetzt, s. Reallexikon der Assyriologie, IV, S. 460b: § 4, und Borger (1988), S. 94, Nr. 134.

23. [Deutsch im Original; A. d. Ü.]

24. Vgl. Bottéro (1973), S. 284-289.

25. Clay (1923), Nr. 12, $36 \mathrm{ff}$.

26. Man beachte diesen in gewissem Sinne abstrakten Satz, der vor ihren konkreten Anwendungen die allgemeine Bedeutung der Vorhersage anzeigt, und vergleiche ihn mit dem in Divination et rationalité untersuchten Dokument (oben Fn. 1), S. 185 ff.

27. Vgl. Bottero (1981).

28. Vgl. Bottéro (1977).

29. Vgl. Annuaire (1974), S. 87-122. 
INDEX

Mots-clés : oniromancie, divination inspirée, divination déductive, oracle, pictogramme onirique

Schlüsselwörter : Traumdeutung, inspirierte Weissagung, deduktive Weissagung, Orakel, Traumbildzeichen

\section{AUTEURS}

\section{JEAN BOTTÉRO}

Jean Bottéro (1914-2007) historien du Moyen-Orient, assyriologue. Nähere Informationen finden Sie hier. 